\title{
A Proposed Model for Traffic Signal Preemption using Global Positioning SYSTEM (GPS)
}

\author{
Nikhil Mascarenhas ${ }^{1}$, Pradeep $\mathrm{G}^{1}$, Manish Agrawal ${ }^{1}$, Subash $\mathrm{P}^{1}$, Ajina $\mathrm{A}^{2}$ \\ ${ }^{1}$ Final year B.E.(Information Science and Engineering, Sir M. Visvesvaraya \\ Institute of Technology, Bangalore. \\ ${ }^{2}$ Faculty, Dept. Of Computer Science and Engineering, Sir M. Visvesvaraya \\ Institute of Technology, Bangalore.
}

\begin{abstract}
A Traffic Signal Preemption system is an automated system that allows normal operation of traffic lights at automated signalized intersections to be preempted. Preemption of signals is generally done to assist emergency vehicles, such as ambulances, so that response times are reduced and right-of-way is provided in a smooth and controlled manner. This paper proposes an innovative and cost-effective server-centric model to facilitate preemption using a simple mobile phone app which uses Global Positioning System (GPS) and a microcontroller which controls traffic signals.
\end{abstract}

\section{KEYWORDS}

Traffic Signal Preemption, GPS, Mobile Application, Arduino Microcontroller, Emergency Vehicle, Road Traffic, Traffic Management.

\section{INTRODUCTION}

Human Life is a valuable asset for a country. Accidents and Medical emergencies such as fire, road accidents, heart attacks etc. occur every day. It is critical for emergency teams to reach the accident spot so as to save human lives. Thus, hospitals and fire stations are spread evenly across the city to reduce response time. However rapid population growth in cities has resulted in high traffic densities on city roads. This is an additional hindrance to fire, ambulance and other emergency vehicles [1].

Conventionally, commuters give way to emergency vehicles, on hearing the vehicle's siren. However since most traffic signals today are automated, an emergency vehicle passing an intersection poses danger to traffic approaching the signal from other roads. Thus an emergency vehicle has to wait for the entire traffic signal cycle to complete in order to pass the intersection. This imposes considerable delay in response time. Other means of transport such as air ambulances are expensive and not feasible for small cities [1].

Thus, Traffic Signal Preemption is the need of the hour, in large cities where traffic densities are ever increasing. Traffic signal Preemption helps in reducing delay and increasing response time and road safety with minimum cost. Several existing technologies use stand-alone hardware to

Sundarapandian et al. (Eds) : ACITY, AIAA, CNSA, DPPR, NeCoM, WeST, DMS, P2PTM, VLSI - 2013 pp. 219-226, 2013. (C) CS \& IT-CSCP 2013

DOI : $10.5121 /$ csit.2013.3423 
achieve this goal. In this paper, we present an innovative and economic solution that uses the accuracy of GPS and the advantages of a server-centric networked system.

The paper is organized as follows. In Section II we discuss existing implementation, the technologies used and their disadvantages. . We discuss the design of the proposed system in Section III. In Section IV the advantages of the proposed system are discussed and in Section V we discuss the future scope and possible enhancements.

\section{SURVEY OF EXISTING IMPLEMENTATIONS}

Traffic preemption devices are implemented in a variety of ways such as acoustic devices, line of sight, and localized radio signals.

Acoustic Systems use audio sensors to detect the pattern of waves from the siren of an emergency vehicle. . An example of an audio sensor is shown in Fig 1. Such a system can either be used independently or it can also be used together with another system. Disadvantage of this system is that traffic preemption can be easily triggered in the wrong direction or at the wrong intersection as sound waves are reflected and traverse in all directions. Another disadvantage is the nonuniformity of waves emitted from different sirens. A malicious illegal preemption can easily be triggered by simulating the audio of a siren. The hardware for sensors and extra circuitry at each intersection is an additional burden [2].

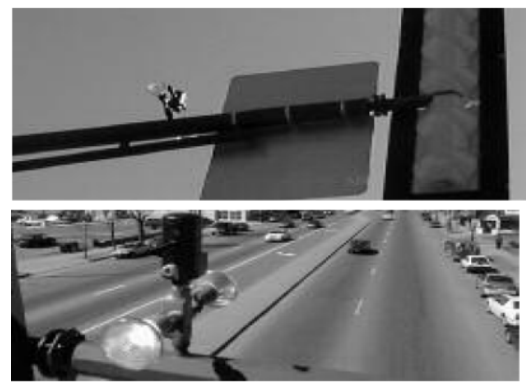

Figure 1. Acoustic Sensors installed at a Traffic Intersection.

Line-of-Sight traffic signal preemption systems emit a fine beam of infrared towards traffic lights in front of the vehicle. Sensors at the intersection (depicted in Fig. 2) detect the beam and change traffic lights accordingly to provide right-of-way to the emergency vehicle. A visible range strobe light may also be used in some cases. Obstructions to the line-of-sight, pollutants and other atmospheric conditions contribute to poor preemption. As with acoustic systems, the cost of additional hardware in both the ambulance and the intersection is high. The system is insecure and hardware to trigger preemption illegally can be easily forged [2].

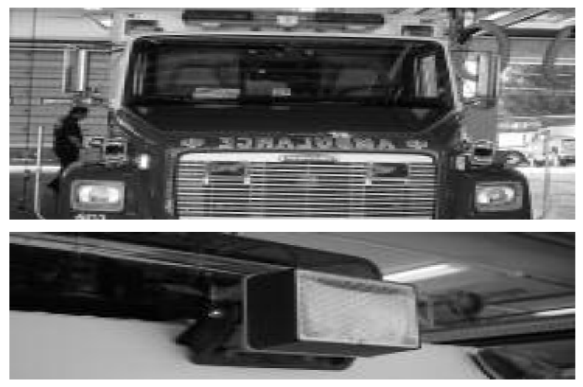

Figure 2. Light-based emitters installed on an Ambulance. 
Radio-based traffic-preemption systems use radio signals to trigger preemption. This overcomes most of the disadvantages of Line-of-sight and Acoustic systems. Radio-signals are not blocked by visual obstructions, lighting or weather conditions. However additional hardware is still a major expense [2]. The model proposed in this paper will make use of GPS technology to overcome all the above limitations. The advantages of using such a system are listed in section IV.

\section{PROPOSED MODEL}

The proposed model will use GPS hardware, already existing in most smart phones, to receive and transmit GPS signals. These signals are transmitted to a central server which can communicate to traffic signal controllers via Internet. The model consists of 3 main components as depicted in Fig. 3.

- An Arduino Microcontroller at each traffic intersection with an Ethernet Shield to enable it to connect to the Internet.

- An Android Application for an Android Smartphone will have to be carried by the staff of each ambulance. This app will receive GPS coordinates and transmit the same to a web server.

- A Web Server which receives GPS coordinates of all emergency vehicles and then sends preemption signals to traffic signal controllers accordingly.

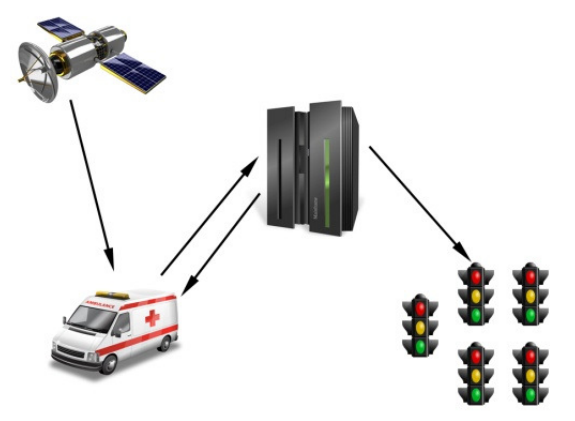

Figure 3. An ambulance (with an Android device) receives GPS coordinates from satellites and then transmits the coordinates to the web server. The web server in turn computes position, speed and other factors and then sends preemption commands to the corresponding traffic signal where the ambulance is approaching.

\subsection{Module 1: Arduino Microcontroller}

Arduino is an Open Source Hardware platform. It consists of a microcontroller development platform which can be programmed and a development environment for creating custom software for the board. Additional capabilities can be provided using add-on boards. All the components including Circuit diagrams and other specification is open-source and is available to modify [3].

In other words Arduino is a tiny microcontroller board with a number of connection sockets that can be connected to external electronic components, such as microphones, relays, light sensors, motors, laser diodes, buzzers etc. You can power an Arduino board either from a computer using a USB cable or from a 9V DC power source. Once the program is uploaded the Arduino board can be disconnected from the computer and can work independently. 


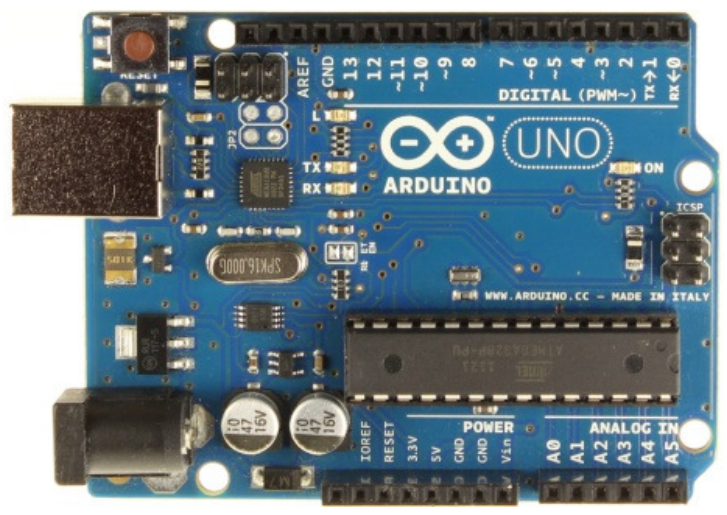

Figure 4. Arduino Uno

Additional capability can be added to the Arduino board by adding auxiliary boards (also known as shields). The shields have pins in the same physical layout as the Arduino board, thus they can simply be mounted on the headers of the Arduino board. The Arduino board controls the shield by accessing the shields pins via the Arduino pins. Arduino provides some programming libraries which allow users to easily include sensors and device in their projects instead of writing programs from scratch. Some examples of Arduino shields are Arduino Ethernet Shield, Wi-Fi Shield etc., that allows the board to connect to the internet and be networked with other devices [4].

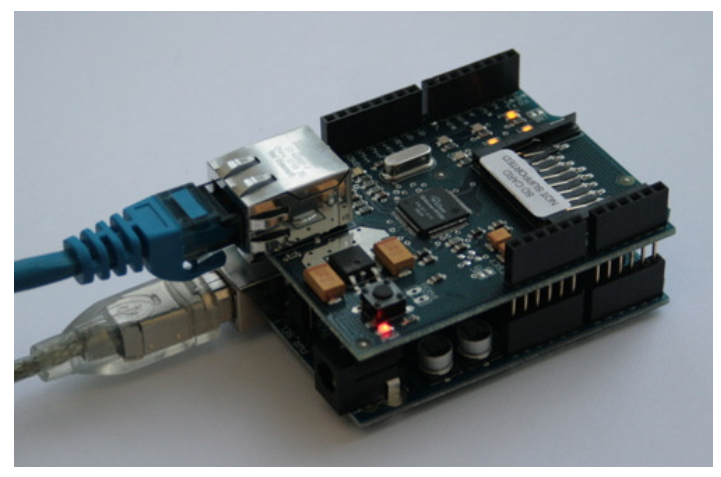

Figure 5. An Arduino Ethernet Shield mounted over an Arduino Uno [4].

In this proposed model, we will be using an Arduino Uno Microcontroller with an Arduino Ethernet Shield that will perform the following functions:

- Normal Mode: Operate the traffic signal lights and buzzer normally, using pre-stored timings in a cyclic manner.

- Accept preemption commands from the web server and switch to Emergency mode smoothly by changing signal lights to the corresponding road.

- Accept return-to-normal mode commands from the web server and switch back to Normal mode. 


\subsection{Module 2: Android App using Location Manager}

Android is a software stack for mobile devices that includes an operating system, middleware and key applications. By providing an open development platform, android offers developers the ability to build extremely rich and innovative applications. Developers have full access to the same framework APIs used by the core applications. The architecture is designed to simplify the reuse of components. Any application can make use of the capabilities of other applications. This same mechanism allows components to be replaced by the user. The Android SDK provides the tools and APIs necessary to begin developing applications on the Android platform [5].

GPS is a space-based satellite navigation system that provides location and time information during all weathers anywhere on or near the earth, where there is an unobstructed line of sight to four or more GPS satellites. Satellites measure accurate time by using an inbuilt atomic clock which are of high-precision. At any given time a GPS receiver possesses information about the position of a given satellite. By timing how long signals take to traverse the distance between the satellite and the receiver, the precise location of the receiver can be computed.

A ground receiver can calculate its $3 \mathrm{D}$ position, that is latitude, longitude and altitude by mapping the data from three different satellites. A fourth satellite may be used to improve accuracy and correct errors in timing offsets between the clock in the satellite and that in the receiver. The more satellites there are, the greater the accuracy is [6]. Android provides 4 classes to help developers make use of inbuilt GPS receivers in smart phones. They are:

- Location Manager

- Location Provider

- Geocoding.

- Google Maps.

The proposed model will use an android application which will be used on a smart phone or tablet installed in every ambulance. The app will use the Location Manager, and Location Provider to perform the following functions:

- The app can only be accessed using a secure login.

- The staff of the emergency vehicle can then select a destination using Google Maps.

- The shortest path to the destination will be displayed.

- The staff can select a priority level and request an emergency status.

- Once the vehicle is declared as an emergency vehicle, the app will send regular updates to the server, which consists of the geo-coordinates of the vehicle, obtained using GPS Location Manager.

\subsection{Module 3: Web Server}

The primary function of a web server is to deliver web pages and files, on request to clients, using the Hypertext Transfer Protocol (HTTP). The web server in the proposed model will perform the following functions: 
- Will accept login authentication requests from android device app.

- Will accept emergency status requests from android app.

- Accepts and monitors geo-coordinates of declared emergency vehicles in a database on the server.

- For each emergency vehicle, the closest approaching traffic signal is computed.

- Preemption commands are sent to the corresponding traffic controller.

- Commands are also sent to the traffic controller, to return to normal mode once the emergency vehicle has passed.

- Maintains logs and statistics of preempted signals, emergency vehicles etc.

\section{AdVantages of The Proposed System}

The proposed system offers several advantages in terms of cost and the simplicity to implement the system.

- By using an android based phone, the cost of implementing hardware on each and every emergency vehicle is greatly reduced. The cost of owning a smart phone is being lowered with newer and more economic models entering the market every week. The phone can be used for other purposes by the hospital or dispatcher to know the status of the patient and other details.

- The Arduino Microcontroller and Ethernet Shield that needs to be installed at every traffic intersections is open source and comes at a market price of about Rs 4000 (appx. $\$ 80$ ). Since the hardware is open source, this cost can be lowered further if customized manufacturing is done in bulk.

- GPS technology has improved a lot in recent years and it retrieves accurate locations. Accurate atomic clocks in GPS satellites provide a way to calculate location that can be precise to the closest millimeter.

- Due to accuracy of GPS there is very little chance of false triggering of preemption. In contrast, Radio and Acoustic based systems can easily trigger preemption in the opposite direction or at an intersection a few feet away.

- The entire system will have all the security benefits of a centralized secure web server. Illegal hardware that can preempt signals cannot be purchased, as these will not be recognized by the central server. In contrast, other systems such as Acoustic, Line of Sight and Radio based implementations do not provide any kind of security against illegal access. A clever analysis can be done and hardware can be forged.

The only demerit of the proposed system is that in densely populated cities, tall buildings may prevent satellite signals in reaching the GPS receiver. This demerit doesn't pose much threat as Android smart phones are capable of computing location based on mobile tower triangulation and also close range Wi-Fi signals.

The benefits and demerits of the proposed model as well as other implementations are tabulated in Table 1. 
Table 1. Comparision Of Technologies.

\begin{tabular}{|l|l|l|l|l|}
\hline Consideration & $\begin{array}{l}\text { Acoustic } \\
\text { System }\end{array}$ & $\begin{array}{l}\text { Line of Sight } \\
\text { System }\end{array}$ & Radio System & $\begin{array}{l}\text { Proposed Model } \\
\text { (GPS) }\end{array}$ \\
\hline $\begin{array}{l}\text { Dedicate Emitter } \\
\text { Required? }\end{array}$ & No & Yes & Yes & $\begin{array}{l}\text { No (smart phone } \\
\text { serves as emitter) }\end{array}$ \\
\hline $\begin{array}{l}\text { Susceptible to } \\
\text { Electronic Noise } \\
\text { Interference? }\end{array}$ & No & No & Yes & No \\
\hline $\begin{array}{l}\text { Clear Line of Sight } \\
\text { Required? }\end{array}$ & Yes & No & No & No \\
\hline $\begin{array}{l}\text { Affected by weather? } \\
\text { No }\end{array}$ & Yes & No & No \\
\hline $\begin{array}{l}\text { Possible Preemption } \\
\text { of Other Approaches }\end{array}$ & Yes & No & Yes & No \\
\hline $\begin{array}{l}\text { Possibility of Illegal } \\
\text { Triggering of } \\
\text { Preemption }\end{array}$ & High & High & High & Low \\
\hline $\begin{array}{l}\text { Centralized Traffic } \\
\text { Signal Monitoring } \\
\text { and Log Statistics }\end{array}$ & No & No & No & Yes \\
\hline
\end{tabular}

\section{CONCLUSION AND FUTURE SCOPE}

The Arduino microcontroller once installed can be used for various other purposes:

- Smart Traffic Management: Using traffic data obtained from various sources such as Google Traffic and Traffic updates from ground controllers, the same server-centric model, using the same microcontroller can be used to slightly manipulate and vary traffic signal timings to ease congested roads.

- The same microcontroller can also be used to display traffic updates on LED screens at traffic signals, so commuters can avoid congested routes.

- Cameras, speed detecting sensors and other sensors can also be connected to the same controller and violations can be viewed automatically at the Central office.

Since the Arduino microcontroller can be used with different types of hardware, this servercentric model using Arduino and GPS can also be used for similar applications such as:

- Automated closing and opening of railway gates.

- Dynamic display of bus arrivals and bus timings at bus stops for passenger convenience.

- Anti-collision systems in railways. 
Our model presents a solution for the easy passage of emergency vehicles by means of preemption of signals at intersections. We plan to implement a small scale working model, and we will demonstrate the network feasibility and feasibility that it can be implemented as a real world application. Traffic Signal Preemption if implemented can reduce emergency response times and increase road safety with minimum inconvenience to regular traffic.

\section{REFERENCES}

[1] Sahar Z Shatnawi, Balqees A Mohammad, Alaa I Kalaf and Hussein R Al-zoubi. A Wireless MobilePhone Approach to Traffic Signal Preemption for Faster Service of Emergency Vehicles. International Journal of Computer Applications 46(3):35-41, May 2012. Published by Foundation of Computer Science, New York, USA.

[2] January 2006, Traffic Signal Preemption for Emergency Vehicles: A Cross- Cutting Study, FHWAJPO-05-010. A report by U.S. Department of Transportation.

[3] Peter J. Gould, Daniel K. Fisher: "Open-Source Hardware Is a Low-Cost Alternative for Scientific Instrumentation and Research". Modern Instrumentation, 2012, 1, 8-20 doi:10.4236/mi.2012.12002 Published Online April 2012 (http://www.SciRP.org/journal/mi). ISSN Print: 2165-9257 ISSN Online: $2165-9273$

[4] Arduino http://www.arduino.cc

[5] Android Developers http://developer.android.com

[6] Jaizki Mendizabal; Roc Berenguer; Juan Melendez (2009). GPS and Galileo. McGraw Hill ISBN 978-0-07-159869-9.

[7] Collura, J. and Willhaus, E. W. 2001. Traffic Signal Preemption and Priority: Technologies, Past Deployments, and System Requirements. Proceedings of the 11th ITS America, Florida, USA.

[8] Vreeken, J., Wiering, M., Veenen, and Koopman, A. 2004 Intelligent Traffic Light Control. Technical report. UU-CS-2004-029.

[9] Global Traffic Technologies, LLC (GTT) http://www.gtt.com.

[10] Lin Dong, Wushan Chen. Real-Time Traffic Signal Timing for Urban Road Multi-intersection. Intelligent Information Management Journal 2010. 21605912

[11] Manav Singhal, Anupam Shukla. Implementation of Location based Services in Android using GPS and Web Services. International Journal of Computer Science Issues 2012. 16940784-16940814.

[12] Karan Mahajan, Manish Mahajan. Navigating the Current Location without the Availability of GPS. International Journal of Engineering and Advanced Technology (IJEAT) ISSN: 2249 - 8958, Volume-2, Issue-3, February 2013. 